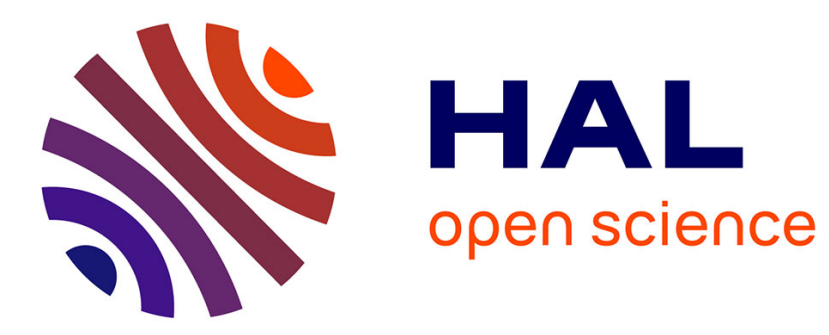

\title{
Discontinuous Coarse Spaces for DD-Methods with Discontinuous Iterates
}

Martin J. Gander, Laurence Halpern, Kévin Santugini-Repiquet

\section{To cite this version:}

Martin J. Gander, Laurence Halpern, Kévin Santugini-Repiquet. Discontinuous Coarse Spaces for DD-Methods with Discontinuous Iterates. 2012. hal-00765821v2

\section{HAL Id: hal-00765821 \\ https://hal.science/hal-00765821v2}

Submitted on 25 Jul 2013

HAL is a multi-disciplinary open access archive for the deposit and dissemination of scientific research documents, whether they are published or not. The documents may come from teaching and research institutions in France or abroad, or from public or private research centers.
L'archive ouverte pluridisciplinaire HAL, est destinée au dépôt et à la diffusion de documents scientifiques de niveau recherche, publiés ou non, émanant des établissements d'enseignement et de recherche français ou étrangers, des laboratoires publics ou privés. 


\title{
Discontinuous Coarse Spaces for DD-Methods with Discontinuous Iterates
}

\author{
Martin J. Gander ${ }^{1}$, Laurence Halpern ${ }^{2}$, and Kévin Santugini Repiquet ${ }^{3}$
}

\section{Introduction}

Basic iterative domain decomposition methods (DDM) can only transmit information between direct neighbors. Such methods never converge in less iterations than the diameter of the connectivity graph between subdomains. Convergence rates are dependent on the number of subdomains, and thus algorithms are not scalable. The use of a coarse space [16] is the only way to provide information from distant subdomains, as they enable global information transfer, ensuring scalability. In this respect, well known methods are the two level additive Schwarz method [3], and the FETI [13] and balancing Neumann-Neumann methods [12, 4, 14]. See also [11] for non-symmetric problems. For complete analyses of such scalable methods, see $[18,17]$.

Adding an effective coarse space correction to an existing method is currently an active area of research, for example in the case of high contrast problems [2, 15]. Combining coarse spaces with methods with discontinuous iterates, such as optimized Schwarz methods (OSM [8]) is also non-trivial, see [6] and chapter 5 in [5] which contain extensive numerical tests, and [7] for a rigorous analysis of a special case. For restricted Additive Schwarz (RAS [1]), which also produces discontinuous global iterates since they are glued from local ones by the $\widetilde{R}$ operators in RAS in an aribirary fashion, see [9] in the present proceedings. We explain in $\$ 2$ why an effective coarse space for non-overlapping OSM (and DDMs with discontinuous iterates in general) should inherently be discontinuous. In $\S 3$, we present one possible realization of a coarse grid correction based on a discontinuous coarse space, and we show that convergence in one coarse correction step can be obtained, although this is only practical in one dimension. For higher dimensional problems, we then propose approximations of this optimal coarse space. In $\S 4$, we present numerical experiments with this new algorithm, and finally give an outlook on future work in $\S 5$.

1. Université de Genève, Section de mathmatiques, e-mail: Martin. Gander@unige.ch

2. Laboratoire Analyse, Géométrie \& Applications UMR 7539 CNRS, Université PARIS 13, 93430

VILLETANEUSE, FRANCE, e-mail: halpern@math.univ-paris13.fr

3. Université Bordeaux, IMB, CNRS UMR5251, MC2, INRIA Bordeaux - Sud-Ouest e-mail:

Kevin.Santugini@math.u-bordeauxl.fr 


\section{Choosing a good coarse space}

In this section, we explain why it makes sense to consider discontinuous coarse space corrections. We place ourselves in a continuous setting and consider the model problem

$$
\eta u-\Delta u=f \quad \text { in } \Omega, \quad \gamma u=0 \quad \text { on } \partial \Omega,
$$

where $\Omega$ is a polygonal domain in $\mathbb{R}^{d}(d \geq 1)$, and $\gamma$ is a trace operator.

Let $\left(\Omega_{i}\right)_{1 \leq i \leq N}$ be a non-overlapping domain decomposition of $\Omega$. A non-overlapping optimized Schwarz method with a coarse grid correction is of the form

\section{Algorithm 1 (Generic).}

1. Initialize $u_{i}^{0}$, either by zero or using the coarse solution.

2. For $n \geq 0$ and until convergence

a. In each subdomain $\Omega_{i}$, compute the uncorrected iterates $u_{i}^{n+1 / 2}$ in parallel using the optimized Schwarz algorithm.

b. Compute a coarse correction $U^{n+1}$ belonging to a coarse space $X$.

c. Set the corrected iterates to $u_{i}^{n+1}:=u_{i}^{n+1 / 2}+U^{n+1}$.

3. Set either $u_{i}:=u_{i}^{n-1 / 2}$ or $u_{i}:=u_{i}^{n}$ where $n$ is the exit index of the above loop.

Instead of explaining in detail how the coarse correction $U^{n+1}$ is computed, we first focus on the more important question of how to choose the coarse space $X$.

\subsection{Suboptimality of a conformal coarse space}

We first explain why with a coarse space $X \subset H^{1}(\Omega)$, it is not possible to compute a very good coarse correction for a domain decomposition method with discontinuous iterates. A function $u$, with $u_{\mid \Omega_{i}}$ in $H^{1}(\Omega)$, is a weak solution of (1) if

1. $u$ satisfies (1) inside each subdomain $\Omega_{i}$,

2. $u$ has no jump between two adjacent subdomains,

3. the normal derivative of $u$ has no jump between two adjacent subdomains.

In an efficient domain decomposition algorithm, each step of the algorithm should improve as many of these three conditions as possible. In particular, the coarse grid correction should be such that the iterates $u_{i}^{n+1}$ are closer to satisfying these three conditions than the uncorrected iterates $u_{i}^{n+1 / 2}$. However:

1. The uncorrected iterates already satisfy the equation inside each subdomain.

2. The uncorrected iterates are discontinuous, they have jumps in the Dirichlet traces along interfaces.

3. The uncorrected iterates have also discontinuous normal derivatives, they have jumps in the Neumann traces along interfaces. 
Using continuous coarse functions is suboptimal for a method that produces discontinuous iterates, since they can not reduce the Dirichlet jumps. Using instead a discontinuous coarse space, for example $P_{0}$, then the Dirichlet jumps can be improved, but not the Neumann jumps. If the coarse functions are even more regular, for example $\mathscr{C}^{1}$ on the whole domain, then neither the Dirichlet jumps nor the Neumann jumps can be improved.

\subsection{Better coarse spaces for methods with discontinuous iterates}

To be effective, a coarse space for a domain decomposition method that produces discontinuous iterates must contain discontinuous functions. Furthermore the discontinuities must be aligned with the interfaces between subdomains. Suppose that the subdomains $\Omega_{i}$ form a conforming polygonal mesh $\mathscr{T}^{\Omega}$ of $\Omega$ (triangles or rectangles in two dimensions). The local polynomial space $\mathscr{P}_{1}$ is $\mathbb{P}_{1}$ in the former case, $\mathbb{Q}_{1}$ in the latter. The conforming coarse space is $\mathscr{P}_{1}\left(\mathscr{T}^{\Omega}\right)=\left\{v \in \mathscr{C}^{0}(\bar{\Omega}), \forall i, v_{\mid \Omega_{i}} \in\right.$ $\left.\mathscr{P}_{1}\right\}$, but a better choice is the discontinuous coarse space (or broken in the Discontinuous Galerkin literature) $\mathscr{P}_{1}^{\text {disc }}\left(\mathscr{T}^{\Omega}\right)=\left\{v, \forall i, v_{\mid \Omega_{i}} \in \mathscr{P}_{1}\right\}$, where the continuity across the interfaces is no longer required.

In addition, for linear problems, it is important for the coarse shape functions to be solutions of the homogeneous counterpart of equation (1) inside each subdomain, because then the corrected iterates are also solutions of the interior equation inside each subdomain. To see this, it suffices to note that the error between the monodomain solution and any iterates produced by the optimized Schwarz method is always a solution to the homogeneous equation inside each subdomain. Therefore, in $H_{0}^{1, d i s c}(\Omega)=\left\{u \in L^{2}(\Omega), \forall i, u_{\mid \Omega_{i}} \in H^{1}\left(\Omega_{i}\right), u=0\right.$ on $\left.\partial \Omega\right\}$, the space

$$
\mathscr{A}=\left\{u \in H_{0}^{1, \text { disc }}(\Omega), \forall i,(\eta-\triangle) u_{\mid \Omega_{i}}=0\right\}
$$

is an ideal candidate for a coarse space. For one-dimensional problems, the space $\mathscr{A}$ is finite dimensional, and can directly be used as the coarse space. In higher dimensions, the space $\mathscr{A}$ is infinite dimensional for the continuous problem, and must therefore be discretized as well to be practical: a finite dimensional subspace of $\mathscr{A}$ must be chosen. To do so, one only needs to choose boundary conditions on each $\partial \Omega_{i}$. For the particular algorithm presented in the next section, the intersection of the coarse space with $H^{1}$ should be "big enough", for there to be enough test functions. This can be guaranteed by constructing coarse elements with potentially compatible Dirichlet conditions.

For these reasons we introduce the space of all discontinuous functions, whose element shape functions are solutions to the homogeneous equation inside each subdomain, with Dirichlet boundary conditions:

$$
\mathscr{P}_{1}^{\mathscr{A}}\left(\mathscr{T}^{\Omega}\right)=\left\{u \in \mathscr{A}, \exists \hat{u} \in \mathscr{P}_{1}^{\mathrm{disc}}\left(\mathscr{T}^{\Omega}\right), u=\hat{u} \text { in } \bigcup_{i=1}^{N} \partial \Omega_{i}\right\}
$$


Remark 1. Other Dirichlet boundary conditions can be used to define the discontinuous coarse elements. Any finite dimensional vector space of continuous functions defined over $\bigcup_{i=1}^{N} \partial \Omega_{i}$ may be used to construct finite dimensional coarse spaces that are subsets of $\mathscr{A}$ with a "big enough" continuous subset.

Now that we have chosen the coarse space, we can design an efficient algorithm to compute a discontinuous coarse space correction. The coarse correction must be chosen such that it diminishes both Dirichlet and Neumann jumps while not losing too much in terms of satisfying the interior equations in each subdomain. Using the full coarse space $\mathscr{A}$ (which is only practical in one dimension), any good algorithm for computing the coarse correction should converge in a single coarse iteration, because the error between the iterates and the exact solution belongs to $\mathscr{A}$. In the next section, we present such an algorithm, the DCS-DMNV algorithm (discontinuous coarse space - Dirichlet minimizer Neumann variational), which is suitable for finite element methods.

\section{The DCS-DMNV algorithm}

We formulate the algorithm with subdomain iterates at the continuous level, with a discrete coarse space.

Let $X_{d}$ be any finite dimensional coarse space, subspace of $H_{0}^{1, \text { disc }}(\Omega)$ (for example $\mathscr{P}_{1}^{\mathscr{A}}\left(\mathscr{T}^{\Omega}\right)$ defined above), and $X_{c}=X_{d} \cap H^{1}(\Omega)$, which will be non-trivial if we use potentially compatible Dirichlet boundary conditions for the coarse elements. We define the positive quadratic form over $H_{0}^{1, d i s c}(\Omega)$ by

$$
q: H_{0}^{1, d i s c}(\Omega) \rightarrow \mathbb{R}^{+}, \quad u \mapsto \sum_{i j} \int_{\partial \Omega_{i} \cap \partial \Omega_{j}}\left|u_{i}-u_{j}\right|^{2} \mathrm{~d} \sigma
$$

Note that the kernel of $q$ is $H^{1}(\Omega)$. The DCS-DMNV algorithm is then given by:

\section{Algorithm 2 (DCS-DMNV).}

1. Initialize $u_{i}^{0}$ by either zero or $u_{\mid \Omega_{i}}^{0}$ where $u^{0}$ is the coarse solution.

2. Until convergence

a. Compute the local iterates $u_{i}^{n+1 / 2} \in H^{1}\left(\Omega_{i}\right)$ in parallel by

$$
\begin{aligned}
\eta u_{i}^{n+1 / 2}-\Delta u_{i}^{n+1 / 2} & =f \quad \text { in } \Omega_{i}, \\
\frac{\partial u_{i}^{n+1 / 2}}{\partial n_{i}}+p u_{i}^{n+1 / 2} & =\frac{\partial u_{j}^{n}}{\partial n_{i}}+p u_{j}^{n} \quad \text { on } \partial \Omega_{i} \cap \Omega_{j}, \\
u_{i}^{n+1 / 2} & =0 \quad \text { on } \partial \Omega_{i} \cap \Omega .
\end{aligned}
$$

b. Define a global $u^{n+1 / 2} \in H_{0}^{1, \text { disc }}(\Omega)$ as $u_{i}^{n+1 / 2}$ in $\Omega_{i}$. Set $U^{n+1}$ as the unique function in $X_{d}$ such that 


$$
q\left(u^{n+1 / 2}+U^{n+1}\right)=\min _{v \in X_{d}} q\left(u^{n+1 / 2}+v\right)
$$

and satisfying

$$
\begin{aligned}
\eta \int_{\Omega} U^{n+1}(x) v(x) \mathrm{d} x & +\int_{\Omega} \nabla U^{n+1}(x) \nabla v(x) \mathrm{d} x \\
& =-\sum_{i, j} \int_{\partial \Omega_{i} \cap \partial \Omega_{j}}\left(\frac{\partial u_{i}^{n+1 / 2}}{\partial n_{i}}+\frac{\partial u_{j}^{n+1 / 2}}{\partial n_{j}}\right) v \mathrm{~d} \sigma
\end{aligned}
$$

for all test functions $v$ in $X_{c}$.

c. Set $u_{i}^{n+1}:=u_{i}^{n+1 / 2}+U^{n+1}$.

3. Set $u:=u_{i}^{n-1 / 2}$ on $\Omega_{i}$ for each $i$ in $\{1, \ldots, N\}$.

Proposition 1 (Existence of the coarse iterate). Let $\left(u_{i}^{n+1 / 2}\right)_{1 \leq i \leq N}$ be the local iterates, . Then there exists a unique $U^{n+1}$ in $X_{d}$ that satisfies (5).

Proof. The function $V \mapsto q\left(u^{n+1 / 2}+V\right)$ is quadratic, choose one minimizer $U_{d}^{n+1}$ on $X_{d}$. By Lax-Milgram's Lemma, there exists a unique $U_{c}^{n+1}$ in $X_{c}$ such that $U^{n+1}=$ $U_{c}^{n+1}+U_{d}^{n+1}$ satisfies (5b). Uniqueness comes from the fact that $q$ is quadratic.

The DCS-DMNV algorithm 2 has the important property of converging in a single coarse step if the full coarse space $\mathscr{A}$ is used. However, it is only practical in a one dimensional setting as the coarse space is too big in higher dimensions. We state that theorem in the discrete case.

Theorem 1 (Convergence in a single coarse step for the full coarse space). Let $\Omega$ be a bounded polygonal domain in $\mathbb{R}^{d}$. Let $\left(\Omega_{i}\right)_{1 \leq i \leq N}$ be a domain decomposition of $\Omega$ that also forms a coarse mesh of $\Omega$. Let $\mathscr{T}_{h}$ be a simplicial or a cartesian fine mesh on $\Omega$ which is a refinement of the $\left(\Omega_{i}\right)_{1 \leq i \leq N}$ domain decomposition. Let $\mathscr{F}$ be the conformal finite element space given either by $P_{1}\left(\mathscr{T}_{h}\right)$ if $\mathscr{T}_{h}$ is simplicial or by $Q_{1}\left(\mathscr{T}_{h}\right)$ if $\mathscr{T}_{h}$ is cartesian. Let $\mathscr{F}^{\text {disc }}$ be the set of all functions on $\Omega$ whose restriction to each $\Omega_{i}$ is also the restriction of a function belonging to $\mathscr{F}$ to $\Omega_{i}, \mathscr{F}_{0}$ be the space of functions in $\mathscr{F}$ vanishing on all subdomain boundaries.

Let $X_{d} \subset \mathscr{F}^{\text {disc }}$ be a coarse space. Suppose all elements in $X_{d}$ satisfy the homogenous variational equation for all test functions in $\mathscr{F}_{0}$. Let $X_{c}=X_{d} \cap \mathscr{F}$. Suppose $u \mapsto\left(\left(u\left(x_{i, j}\right)\right)_{1 \leq j \leq k_{i}}\right)_{1 \leq i \leq N}$ is from $X_{d}$ onto $\prod_{i=1}^{N} \mathbb{R}^{k_{i}}$ where $k_{i}$ is the number of nodes of $\mathscr{T}_{h}$ located on $\partial \Omega_{i} \backslash \partial \Omega$ and where $x_{i, j}$ is the $j$-th node located on $\partial \Omega_{i} \backslash \partial \Omega$. Then, for any choice of initial fine iterate $\left(u_{i}^{0}\right)_{1 \leq i \leq N}$ satisfying the variational equation for all test functions in $\mathscr{F}_{0}$, the DCS-DMNV algorithm 2 converges in a single coarse step.

Proof. Let $\left(U_{i}\right)_{1 \leq N}$ be the coarse correction. Let $u_{i}^{1}=u_{i}^{0}+U_{i}$ be the corrected iterates. The corrected iterates must satisfy the minimum jump condition (5a). Since $u \mapsto\left(\left(u\left(x_{i, j}\right)\right)_{1 \leq j \leq k_{i}}\right)_{1 \leq i \leq N}$ is onto, it is possible to completely cancel the jumps, therefore $q\left(\left(u_{i}^{1}\right)_{1 \leq i \leq N}\right)=0$ and $u^{1}$ defined over $\Omega$ as $u_{\mid \Omega_{i}}^{1}=u_{i}^{1}$ belongs to $\mathscr{F}$, i.e. 

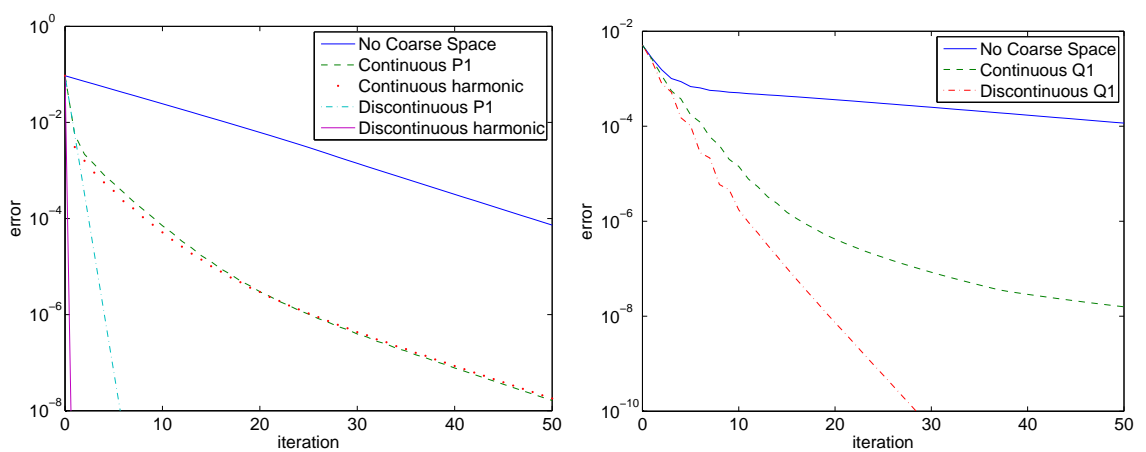

Fig. 1 Convergence curves of the DCS-DMNV algorithm in $1 D$ (left) and $2 D$ (right)

is continuous across subdomains. Moreover, since the coarse correction satisfies the homogenous counterpart of (1) inside each subdomain, the corrected iterates satisfy the variational equation for all test functions in $\mathscr{F}_{0}$. By $(5 b)$, the corrected iterates also satisfy the variational equation for all test functions $v$ in $X_{c}$. Since $u \mapsto\left(\left(u\left(x_{i, j}\right)\right)_{1 \leq j \leq k_{i}}\right)_{1 \leq i \leq N}$ is onto, $\mathscr{F}=X_{c}+\mathscr{F}_{0}$. Therefore, the corrected iterates satisfy the variational equation for all test functions in $\mathscr{F}$.

In a practical implementation however, convergence in a single coarse iteration would only be possible if the coarse space contains all the degrees of freedom on the interfaces corresponding to the fine discretization of the subdomain problems, which would be a very rich and expensive coarse space. We will see in the next section that a linear approximation of all the degrees of freedom on the interfaces already leads to a very good discontinuous coarse correction.

\section{Numerical results}

We implemented the DCS-DMNV algorithm 2 in one and two dimensions, using a finite element discretization based on a regular cartesian grid. In $1 D$, we chose $\Omega=] 0,4[, \eta=10$ and the right hand-side $f(x)=-1$. For the Robin parameter, we used $p=5$, with 60 subdomains. Convergence curves are presented in Figure 1.

As expected, a coarse grid correction with conforming $\mathbb{P}_{1}$ finite elements already improves convergence. Requiring the coarse shape functions to be solutions of the homogeneous equation within each subdomain does not bring any further gain. A striking improvement is the use of discontinuous $\mathbb{P}_{1}$ elements. Optimal convergence (see Theorem 1) can then be reached if in addition the coarse functions solve the homogeneous equation inside each subdomain.

In two-dimension, we chose $\eta=0$ and iterate directly on the error equations, i.e., we solve $-\Delta e=0$ but start with random boundary conditions on each subdomain. $Q_{1}$ elements discretize $\left.\Omega=\right] 0,4[2$, and the algorithm is run with $p=12.4$, 

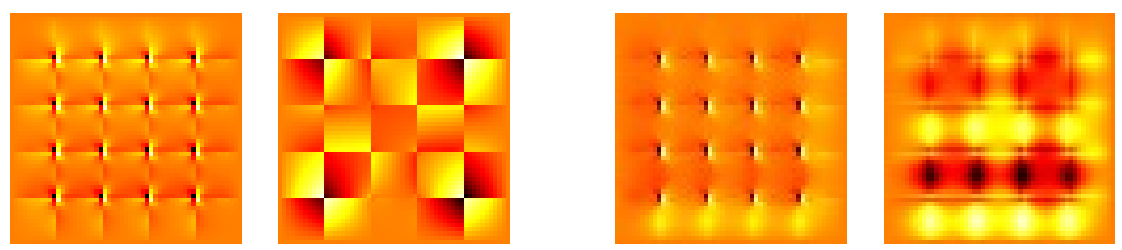

Fig. 2 Error of the algorithm with continuous (left) and discontinuous (right) coarse grid correction at iterations 5 and 20. Each error has been renormalized independently.

$5 \times 5$ subdomains and $10 \times 10$ cells per subdomain. It is important for the Robin boundary conditions to be lumped, see [10]. To compute the coarse correction, we use the Conjugate Gradient algorithm to compute the result of the multiplication of the pseudo-inverse of $Q, q(u, v)=(Q u \mid v)$ with a right hand-side derived from the uncorrected iterates. This gives us one minimizer in $X_{d}$ of the $q$ functionnal. To satisfy the variational condition, an additional continuous coarse correction can then be computed in $X_{c}$.

As in the $1 D$ case, the convergence curves presented in Figure 1 show that the discontinuous coarse space correction leads to a much faster convergence than the continuous one. Even though the discontinuous coarse space is only a subset of the optimal theoretical coarse space, the improvement over continuous coarse spaces is substantial. In order to see in the error how the jumps slow down the convergence of the continuous coarse correction version, we present in Figure 2 a few snapshots of the errors. We observe the formation of a checkerboard like structure which cannot be corrected by a continuous coarse space. Once the errors look like a checkerboard, the convergence of the continuous coarse correction algorithm slows down considerably. Using a discontinuous coarse space prevents the checkerboard like structure from appearing.

\section{Conclusion}

We have shown that for domain decomposition methods with discontinuous iterates, the use of a discontinuous coarse space greatly improves that of a standard continuous one. We have designed one such discontinuous coarse space algorithm, the DCS-DMNV algorithm, the formulation of which is well suited for finite element discretizations. In practice, this algorithm should be used in conjunction with Krylov acceleration. We intend to study the behavior of the Krylov accelerated DCSDMNV in a forthcoming paper. We are currently studying such algorithms also for finite difference and finite volumes schemes, and investigating how the optimization parameter $p$ in the transmission conditions interacts with the Dirichlet boundary conditions used in the definition of the coarse space. 


\section{References}

1. Cai, X.C., Sarkis, M.: A restricted additive Schwarz preconditioner for general sparse linear systems. SIAM Journal on Scientific Computing 21, 239-247 (1999)

2. Dolean, V., Nataf, F., Scheichl, R., Spillane, N.: Analysis of a two-level schwarz method with coarse spaces based on local dirichlet to neumann maps. Computational Methods in Applied Mathematics 12(4), 391-414 (2012). DOI 10.2478/cmam-2012-0027

3. Dryja, M., Widlund, O.B.: An additive variant of the Schwarz alternating method for the case of many subregions. Tech. Rep. 339, also Ultracomputer Note 131, Department of Computer Science, Courant Institute (1987)

4. Dryja, M., Widlund, O.B.: Schwarz methods of Neumann-Neumann type for threedimensional elliptic finite element problems. Comm. Pure Appl. Math. 48(2), 121-155 (1995)

5. Dubois, O.: Optimized Schwarz methods for the advection-diffusion equation and for problems with discontinuous coefficients. Ph.D. thesis, McGill University (2007)

6. Dubois, O., Gander, M.J.: Convergence behavior of a two-level optimized Schwarz preconditioner. In: Domain Decomposition Methods in Science and Engineering XXI. Springer LNCSE (2009)

7. Dubois, O., Gander, M.J., Loisel, S., St-Cyr, A., Szyld, D.: The optimized Schwarz method with a coarse grid correction. SIAM J. on Sci. Comp. 34(1), A421-A458 (2012)

8. Gander, M.J.: Optimized Schwarz methods. SIAM J. Numer. Anal. 44(2), 699-731 (2006)

9. Gander, M.J., Halpern, L., Santugini, K.: A new coarse grid correction for RAS. In: Domain Decomposition Methods in Science and Engineering XXI. Springer LNCSE (2013). Submitted

10. Gander, M.J., Hubert, F., Krell, S.: Optimized Schwarz algorithm in the framework of DDFV schemes. In: Domain Decomposition Methods in Science and Engineering XXI. Springer LNCSE (2013). Submitted

11. Japhet, C., Nataf, F., Roux, F.X.: Extension of a coarse grid preconditioner to non-symmetric problems. In: J. Mandel, C. Farhat, X.C. Cai (eds.) Domain decomposition methods, 10 (Boulder, CO, 1997), Contemporary Mathematics, vol. 218, pp. 279-286. Amer. Math. Soc., Providence, RI (1998)

12. Mandel, J.: Balancing domain decomposition. Communications in Numerical Methods in Engineering 9(3), 233-241 (1993). DOI 10.1002/cnm.1640090307

13. Mandel, J., Brezina, M.: Balancing domain decomposition for problems with large jumps in coefficients. Math. Comp. 65, 1387-1401 (1996)

14. Mandel, J., Tezaur, R.: Convergence of a substructuring method with Lagrange multipliers. Numer. Math. 73, 473-487 (1996)

15. Nataf, F., Xiang, H., Dolean, V., Spillane, N.: A coarse sparse construction based on local Dirichlet-to-Neumann maps. SIAM J. Sci. Comput. 33(4), 1623-1642 (2011)

16. Nicolaides, R.A.: Deflation conjugate gradients with application to boundary value problems. SIAM J. Num. An, 24(2), 355-365 (1987). DOI doi:10.1137/0724027

17. Smith, B.F., Bjørstad, P.E., Gropp, W.: Domain Decomposition: Parallel Multilevel Methods for Elliptic Partial Differential Equations. Cambridge University Press (1996)

18. Toselli, A., Widlund, O.: Domain Decomposition Methods - Algorithms and Theory, Springer Series in Computational Mathematics, vol. 34. Springer (2004) 